\title{
FREQUENCY CHARACTERISTICS OF SEISMIC PIEZOELECTRIC SENSORS UNDER ONE-DIMENSIONAL MECHANICAL ACTION
}

\author{
Nikolay Georgiev ${ }^{1}$, YAVOR Boichev ${ }^{1 *}$, Konstantina \\ BELOTELOVA $^{2}$, IVAN IVANOV ${ }^{1}$ \\ ${ }^{1}$ Institute of Metal Science, Equipment and Technologies with Hydro- and \\ Aerodynamics Centre "Acad. A. Balevski", Bulgarian Academy of Sciences \\ (IMSETHC-BAS), Sofia, Bulgaria \\ ${ }^{2} R$-sensors, Moskow, Russia
}

[Received: 12 February 2020. Accepted: 30 April 2020]

doi: 10.7546/JTAM.50.20.02.03

\begin{abstract}
In this study, a mathematical model that describes the frequency response of piezoelectric sensors under one-dimensional mechanical stress is presented. In laboratory conditions, experimental studies of sensors designed and manufactured by IMSTCHA-BAS and NSF were conducted. These studies confirm the adequacy of the presented mathematical model.
\end{abstract}

KEY WORDS: seismic sensor, piezoelectric sensor, frequency response characteristic, mathematical model.

\section{INTRODUCTION}

Over the last decade, there has been a significant increase in the use of various types of seismic sensors in many fields of science and manufacturing, in systems for measurement, diagnostics and control, in monitoring and security systems, etc. [1-11]. This has come as a result of the increasingly sophisticated technological capabilities for seismic sensor design tailored to various specific needs and of the development of communication and information tools for disseminating and visualizing the data received. Furthermore, the technologies for processing and analyzing primary information received from seismic sensors have been considerably refined. All of this makes it extremely important to gain better knowledge of the seismic sensors frequency response characteristics (FRC).

As is well known, seismic sensors are typically used in two types of systems. One of them can provisionally be called a measuring system, in which the sensor is intended to determine the parameters of the seismic oscillations [11-15]. The seismic sensors used in this system must have flat or slowly changing frequency response curves in the bandwidth where the characteristics of the oscillations of interest are to

\footnotetext{
${ }^{*}$ Corresponding author e-mail: y_boichev@abv.bg
} 
be determined. The second type of system, which can also be provisionally called detector, is intended to detect specific seismic signals whose FRC will reach their maximum in the frequency range where the major portion of the spectrum of seismic oscillations under detection is expected to be located.

The actual frequency response of real seismic sensors depends on a number of factors, some of which can change within certain limits in the process of their production. It is therefore of theoretical and applied interest to find models describing the frequency response of seismic sensors and to determine approaches for their practical use. It is appropriate to evaluate how the models conform with the actual processes, initially for one-dimensional mechanical effects, and only after that for cases where these effects are two- and three-dimensional.

\section{THEORY}

One of the most common types of seismic sensors includes those using the piezoelectric effect. A number of literary sources use a simplified mechanical model of this type of sensor presenting it as a piezoelectric element connected on one of its sides with a base (mounting component and sensor body) and on the other with a weight (most often made of lead), called the inertial element of the sensor. A simplified mechanical diagram of this type of sensor, convenient for modeling one-dimensional mechanical effects, is shown in Fig. 1 [11,14].

The designations used in Fig. 1 are as follows:

- $m_{s}$ is the mass of the inertial element of the sensor (inertial or seismic mass);

- $k$ is the equivalent hardness of the piezoelectric element. It determines the bending and the mechanical deformation of the piezoelectric element due to the difference between the applied force on the sensor body and the inertia of

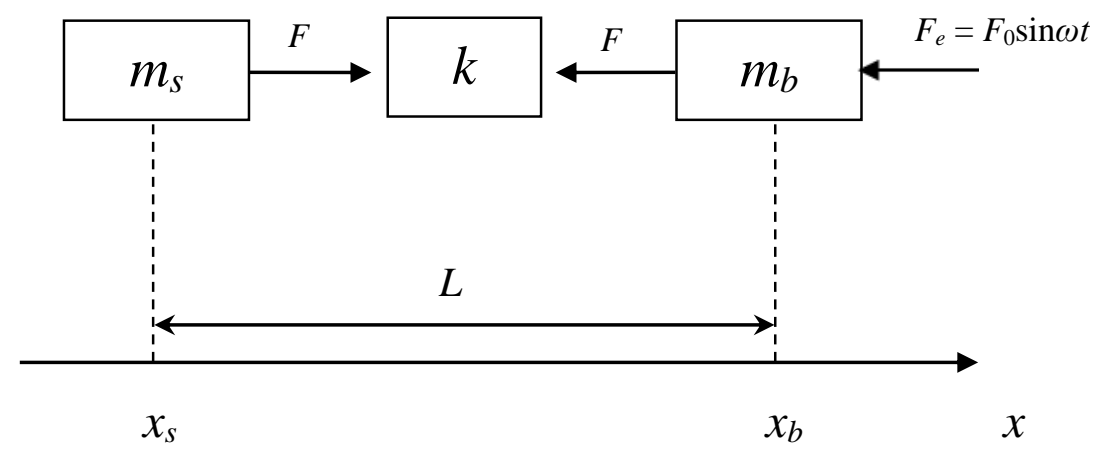

Fig. 1: Simplified mechanical diagram of a seismic sensor. 
the inertial element. It depends on the element's size, shape and the properties of the material of the element;

- $m_{b}$ is the mass on the base (body) of the sensor;

$-F_{e}$ is harmonic coertive force;

- $F$ is the force acting on the piezoelectric element which is generally proportional to the force of the seismic oscillation;

- $x_{s}$ is the coordinate of the inertial element of the sensor;

- $x_{b}$ is the coordinate of the base of the sensor.

Assuming that the piezoelectric element is the elastic component of the sensor's mechanical diagram, the force acting on it can be determined by the relation

$$
\left.F=k\left(x_{s}-x_{b}-L\right)=k\left[\left(x_{s}-x_{b}\right)-L\right)\right]=k\left(d x_{s b}-L\right),
$$

where $L$ is the distance between the inertial element and the base at rest (at rest $x_{s}-x_{b}=L$ and $F=0$ ), and $d x_{s b}=x_{s}-x_{b}$ is the deformation of the elastic material, i.e. the size of the piezoelectric element bending, contracting or stretching.

Assuming that the value of the signal generated by the piezoelectric element $S(f)$ is directly proportional to the forces acting on it, the quoted model describes the frequency response of the seismic sensor by Eq. (2) [11]

$$
S(f)=-r F_{0} /\left(m_{b}\left|4 \pi^{2} f_{s}^{2}-4 \pi^{2} f^{2}\right|\right),
$$

where $F_{0}$ is the amplitude of the seismic vibration force at the point of dislocation of the sensor; $f_{s}$ is the natural resonant frequency of the oscillating system of the sensor; $\|$ is the absolute value of $x$; and $r$ is a conversion factor of the mechanical into electrical oscillation for the particular piezoelectric element.

It is known that the relationship between the natural resonant frequency of the oscillating system, the mass of the oscillating component (usually equal to the mass of the inertial element) and the hardness of the elastic element through which it is connected, is determined by Eq. (3) [11]

$$
k /\left(m_{s}+m_{b}\right)=\left(2 \pi f_{s}\right)^{2} ; \quad \text { or, if } m_{s} \ll m_{b}, \text { then } k / m_{s} \approx\left(2 \pi f_{s}\right)^{2} .
$$

From Eq. (2) it follows that:

$$
\begin{aligned}
& S(f)=\gamma /\left|1-f^{2} / f_{s}^{2}\right|, \quad \text { where, for a particular sensor : } \\
& \gamma=-\left(r F_{0}\right) /\left(4 \pi^{2} f_{s}^{2} m_{b}\right)=\text { const. }
\end{aligned}
$$

The model presented above is relatively simple, but it has the following limitations: 
— it is one-dimensional, representing the vibration of a single-mass system moving in only one direction, whereas the external forces acting on the seismic sensor are actually three-dimensional. In practice, the oscillating component of the seismic sensor moves in two or three directions. Depending on the type of seismic wave (deep or surface), as well as on the type and location of the sensor, it is possible to ignore the influence of the external force along one, but very rarely, on both spatial coordinates;

- the model assumes that the connections between the individual components are perfectly firm and does not take into account the damping of the oscillating system;

- the model does not take into account the nonlinearity and the maximum values constraints of the processes of transformation of mechanical forces into electrical ones. It assumes a unique dependence between the mechanical displacement of the elastic element and the value of the electrical signal generated by the piezoelectric element. Furthermore, the model does not introduce energy constraints, so inadequate results can be expected when the external force frequency approaches the resonant frequency of the oscillating component.

For these reasons, the authors of this model, as well as numerous other studies recommend determining the sensors frequency response experimentally in accredited laboratories.

If the model presented above is adequate, i.e. if it describes relatively well the change in the seismic sensors frequency response, it could be used in the process of their design, and in particular for the prediction of some basic physico-mechanical parameters that could provide the desired type of their frequency response.

In addition, an adequate mathematical model can be used to evaluate the possibilities for practical use of seismic sensors of the same type, such as those in one and the same batch, as well as for quality control in the process of their production and exploitation.

In order to determine the adequacy of the above model to specific types of seismic sensors of the class under consideration, instead of a detailed listing of their physicalmechanical and frequency response characteristics, it is possible to determine their resonant frequency $f_{s}$ and the value of their coefficient $\gamma$ using experimental data.

The calculation of the value of $\gamma$ according to Eq. (5) is dependent on knowing the exact values of $r, F_{0}, f_{s}$, and $m_{b}$, which requires additional precision measurements. It is therefore appropriate to find ways to estimate the coefficient $\gamma$ relatively quickly and based on a small number of measurements. One of the options is to use Eq. (4) 
from which it follows that:

$$
\gamma=S(f)\left|1-f^{2} / f_{s}^{2}\right| .
$$

If the model is adequate, then the relationships indicated by the above formulas must be valid, and as been already stated, $\gamma$ must be constant [11-15]. Therefore, the adequacy of the quoted model to a real seismic sensor can be verified by experimentally measuring the values $S\left(f_{i}\right)$ of the sensor output signals for several different values of the frequencies of the external influences $f_{i}$ and, if possible, by verifying the accuracy of Eqs. (2-(6). In addition, Eq. (6) makes it possible to calculate the partial values of the coefficient $\gamma_{i}$. So, if the model is completely adequate, the values of $\gamma_{i}$ for the different frequencies should be the same or very similar. Taking into account the measurement errors, in an adequate model the values of $\gamma$ should be close to each other and not significantly different from the experimentally determined average value of $\gamma$ (denoted here by $\left.\gamma_{\exp }\right)$, which can be defined as

$$
\gamma_{\exp }=\left(\Sigma \gamma_{i}\right) / n,
$$

where the sum is for values of $i=1 \div n$ and $n$ is the total number of the different frequencies for which measurements were made, and $\gamma_{i}=S\left(f_{i}\right)\left|1-f i^{2} / f^{2} s\right|$.

Using the proposed approach requires knowing only the resonant frequency of the oscillating system $f_{s}$ and preserving the conditions under which the measurements of $S\left(f_{i}\right)$ were taken.

\section{EXPERIMENTAL RESULTS AND DisCUSSIONS}

The adequacy of the cited model for samples of four types of piezoelectric seismic sensors was evaluated at IMSETHC-BAS. This assessment is a necessary condition in applying the model for the analysis of the possibilities for utilizing the sensors in various situations, as well as for the implementation of the project "Study of the amplitude - frequency characteristics of seismic signals generated by surface sources," funded by the Bulgarian Research Fund (FNI).

The experiments were carried out at the Laboratory of Experimental Mechanics at IMSETHC-BAS - Sofia, and included measuring the frequency response characteristics of several types of piezoelectric sensors. For this purpose a BRUEL \& KJAER accelerometer type 4370 No 984125 and an amplifier type 2625 manufactured by the same company were used. Also used was some of the equipment supplied for completing Work Package 2, "Intelligent Security Systems", of Project BG05M2OP0011.002-0006, "Establishment and development of a competence center for quantum communication, intelligent security systems and risk management (Quasar)," which received funding from the European Regional Development Fund through the Operational Programme "Science and Education for Smart Growth, 2014-2020". 
The seismic sensors under consideration were developed by the Institute and are intended to record signals from surface sources, mainly humans and vehicles. They have a cylindrical housing mounted in which, using the corresponding mounting components, are a piezoelectric element, consisting of a pair of metal plates coated with a piezoceramic material, and a lead inertial element.

The seismic sensors under investigation were of the following types:

- Type 11 (T11) - aluminum body, plate thickness of $0.3 \mathrm{~mm}$ and mass of the inertial element 40 grams;

- Type 22 (T22) - aluminum body, plate thickness of $0.2 \mathrm{~mm}$ and mass of the inertial element 115 grams;

- Type 33 (T33) - aluminum body, plate thickness of $0.3 \mathrm{~mm}$ and mass of the inertial element 115 grams;

- Type 55 (T55) - brass body, plate thickness of $0.2 \mathrm{~mm}$ and mass of the inertial element 115 grams.

Table 1 presents the results of the measurement of the values of $S\left(f_{i}\right)$ for the each of the above sensor types. The measured natural resonant frequencies of the oscillating system of the sensors are as follows: for T11 $-f_{s 11}=255 \mathrm{~Hz}$; for T22 $f_{s 22}=115 \mathrm{~Hz}$; for T33 $-f_{s 33}=145 \mathrm{~Hz}$; and for T55 $-f_{s 55}=170 \mathrm{~Hz}$.

It is proposed that the validation of the adequacy of the cited model of the frequency response should be performed according to the following three criteria:

- adequacy of modeling the influence of the mass of the inertial element on the resonant frequencies of the sensors oscillating systems;

- adequacy of modeling the influence of equivalent piezoelectric hardness on the resonant frequencies of the sensors oscillating systems;

- constant value of the coefficient $\gamma$.

In order to evaluate the adequacy of the model using the indicator related to the influence of inertial masses on the resonant frequencies of the sensors oscillating systems, it is appropriate to compare the results for sensors T11 and T33. These sensors are of the same type and, except for manufacturing tolerances, differ only in the value of the masses of their inertial elements. To assess whether the model adequately reflects the influence of the value of inertial masses on the resonant frequencies of the oscillating sensor systems, Eq. (2) can be used. According to it, the relationship between the resonant frequencies and the value of the inertial mass is

$$
f_{s} \approx\left(k / m_{s}\right)^{0.5} / 2 \pi .
$$


Table 1: Measured values of $S\left(f_{i}\right)$

\begin{tabular}{ccccc}
\hline \hline$f_{i}$ & $S\left(f_{i}\right)$ & $S\left(f_{i}\right)$ & $S\left(f_{i}\right)$ & $S\left(f_{i}\right)$ \\
{$[\mathrm{Hz}]$} & 11 & 22 & 33 & 55 \\
\hline 40 & 260 & 300 & 730 & 96 \\
60 & 260 & 370 & 870 & 106 \\
80 & 270 & 560 & 1050 & 120 \\
100 & 280 & 1700 & 1400 & 160 \\
120 & 310 & 1500 & 2800 & 225 \\
140 & 360 & 480 & 10000 & 550 \\
160 & 360 & 320 & 4200 & 1050 \\
180 & 420 & 180 & 1300 & 320 \\
200 & 460 & 130 & 820 & 160 \\
220 & 630 & 100 & 570 & 100 \\
240 & 920 & & & \\
260 & 3400 & & & \\
280 & 2500 & & & \\
300 & 1350 & & & \\
\hline \hline
\end{tabular}

Because T11 and T33 are made of the same piezoelectric elements with the same values of their equivalent hardness $k$, if the model under study is adequate for these sensors, the following dependence must apply:

$$
f_{s 11} / f_{s 33}=\left(m_{s 33} / m_{s 11}\right)^{0.5} .
$$

According to the results of the measurements

$$
\begin{gathered}
f_{s 11} / f_{s 33}=255 / 145 \approx 1.76 ; \\
\left(m_{s 33} / m_{s 11}\right)^{0.5}=(115 / 40)^{0.5} \approx 1.7 .
\end{gathered}
$$

Equation (11) represents the ratio between the resonant frequencies $f_{s 11}$ and $f_{s 33}$ in case the model under study is adequate, and Eq. (10) shows the ratio of the measured values of these resonant frequencies. It can be seen that the difference between the data obtained from Fqs. (10) and (11) is acceptable from a practical point of view. Therefore, it can be assumed that the quoted model adequately reflects the influence of the masses of the inertial elements of the investigated seismic sensors on the resonant frequencies of their oscillating systems.

In order to evaluate the adequacy of the model using the indicator related to the influence of the coefficient $k$ on the resonant frequencies of the sensors oscillating systems, it is appropriate to compare the results for sensors T22 and T33. These 
sensors are of the same type, except for manufacturing tolerances, and differ only in the thickness of the plates on which the piezoelectric coatings are applied and therefore in the value of their inertial masses. Taking into account the physical processes occurring in the seismic sensors under study, it can be assumed that the equivalent hardness $k$ of the piezo element is uniquely related to the thickness of their plates. If we denote the hardness of the plates for the sensors T33 and T22 by $k_{33}$ and $k_{22}$ respectively, then, if the cited model is adequate, the following relationship must be valid in accordance with Eq. (3):

$$
f_{s 33} / f_{s 22}=\left(k_{33} / k_{22}\right)^{0.5} .
$$

Assuming that $k_{33} / k_{22}$ is proportional to the thickness of the respective plates, i.e. to $0.3 / 0.2$, we get

$$
\begin{aligned}
f_{s 33} / f_{s 22} & =145 / 115 \approx 1.26, \\
\left(k_{33} / k_{22}\right)^{0.5} & =(0.3 / 0.2)^{0.5} \approx 1.22 .
\end{aligned}
$$

It is again apparent that the difference between the data obtained from Eqs. (13) and (14) is acceptable from a practical point of view, i.e. it can be assumed that the model presented here adequately reflects the influence of the equivalent hardness of the piezo elements of the seismic sensors under investigation on the resonant frequencies of their oscillating systems.

Of interest are the differences between the resonance frequencies of the T22 and T55 sensors, which share the same parameters but have aluminum and a brass body, respectively.

The relation between these two frequencies is

$$
f_{s 55} / f_{s 22}=170 / 115 \approx 1.478 .
$$

At the same time, the square root of the ratio between the modulus of elasticity of copper, which is the main component of the brass body (between 110 and 128 gigapascals, but due to the admixture with zinc, the upper limit can be assumed) and aluminum ( 70 gigapascals) is

$$
(128 / 70)^{0.5} \approx 1.35 \text {. }
$$

Therefore, it can be seen that the cited model is adequate for taking into account the influence of the change in the elasticity of the body to which the piezoelectric element is connected on the elasticity (i.e., the stiffness) of the body-piezoelectric system and therefore on the resonant frequency of the sensor oscillating system. 
Table 2: Calculated values of $\gamma_{i}$

\begin{tabular}{ccccc}
\hline \hline$f_{i}$ & $\gamma_{i}$ & $\gamma_{i}$ & $\gamma_{i}$ & $\gamma_{i}$ \\
{$[\mathrm{~Hz}]$} & 11 & 22 & 33 & 55 \\
\hline 40 & 253,60 & 263,71 & 674,45 & 90,69 \\
60 & 245,60 & 269,28 & 721,03 & 92,80 \\
80 & 243,43 & 289 & 730,38 & 93,43 \\
100 & 236,94 & 414,56 & 734,13 & 104,64 \\
120 & 241,35 & 133,27 & 882,28 & 112,89 \\
140 & 251,49 & 231,38 & 677,76 & 176,99 \\
160 & 218,27 & 299,43 & 913,91 & 119,90 \\
180 & 210,73 & 260,98 & 703,33 & 38,75 \\
200 & 177,03 & 263,19 & 740,05 & 61,45 \\
220 & 161,07 & 265,97 & 742,15 & 67,47 \\
240 & 105,05 & & & \\
260 & 134,64 & & & \\
280 & 514,23 & & & \\
300 & 518,51 & & & \\
$\gamma_{\text {exp }}$ & 250,85 & 269,08 & 751,95 & 95,9 \\
\hline \hline
\end{tabular}

To assess the adequacy of the model using the indicator associated with the constant value of the coefficient $\gamma$ according to Eq. (7), the values of $\gamma_{i}$ and $\gamma_{\text {exp }}$, which are presented in Table 2, were calculated.

Figure 2 shows the graphs of the change for different frequencies in the values of $\gamma_{i}$ for the four sensors tested, with one unit on the abscissa axis corresponding to $25 \mathrm{~Hz}$.

Table 2 and Fig. 2 show that for the whole frequency range according to the three criteria - "constant value of $\gamma$ ", "insignificant deviation of values of $\gamma_{i}$ against each other" and "insignificant deviation of values of $\gamma_{i}$ relative to $\gamma_{\text {exp }}$ ", the quoted model is inadequate for all of the seismic sensors studied. At the same time, it can be seen that while at frequencies close to the resonance abrupt changes in $\gamma_{i}$ are observed, in the more distant frequency ranges the values of $\gamma_{i}$ are relatively the same and that these two criteria show the adequacy of the model. It is also seen that the mean values of $\gamma_{i}$ for the frequencies lower than the resonant one are different from those for the frequencies higher than the resonant frequency.

On this basis, it can be assumed that for the seismic sensors studied here the cited model is adequate only for frequencies different from the resonant ones by about $10 \%$ (for frequencies higher than the resonant ones) or by up to $20 \%$ (for frequencies lower than the resonant ones), i.e. if the seismic sensors are intended for use 


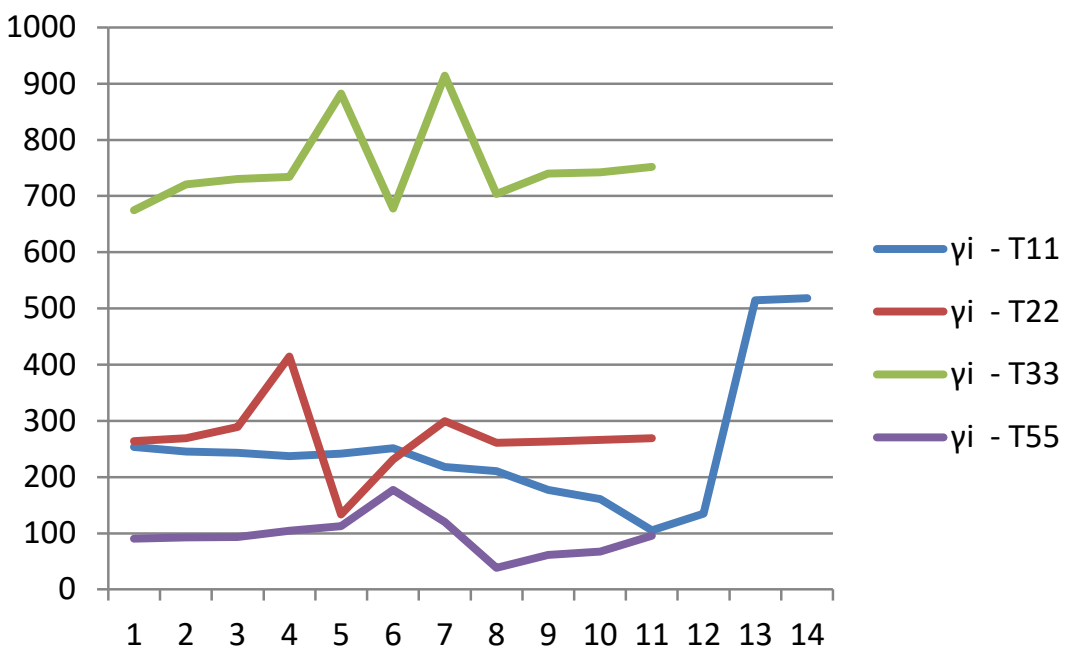

Fig. 2: Values of $\gamma_{i}$ for the four sensors.

in the so-called measurement systems. In the frequency range close to the resonance frequency, i.e. if the seismic sensors are intended for use in the so-called detector systems, their frequency response should be modeled using other types of mathematical dependencies.

\section{CONCLUSIONS}

1. The mathematical model of the frequency response of seismic sensors represented by Fqs. ( 2 and 4 ) can be used to model one-dimensional mechanical impacts on seismic sensors of the studied types in frequency ranges different from the resonant one by about $10 \%$ (for frequencies higher than the resonant) or by up to $20 \%$ (for frequencies below the resonant). This indicates that the model is convenient when the investigated sensors are used in the so-called measurement systems.

2. When designing seismic sensors of the studied type using Eq. (3), it is possible to predict the influence of the inertial mass used and/or of the stiffness of the elastic material on their resonant frequencies.

3. Changing the thickness of the plates or of the body of the seismic sensor results in a corresponding change in the hardness factor of the elastic system and a change in its resonance frequency. This suggests that the possible damping of seismic vibrations coming from the ground where the sensor is placed may also affect its frequency response. The latter assumption needs to be proven, but if true, special measures should be taken when burying seismic sensors in the ground.

4. In the frequency range away from the resonant frequency, the sensors under 
study have relatively uniform frequency response, i.e. in these areas, they can be used to measure the spectrum of seismic oscillations from different surface sources. In order to obtain better results at low frequency measurements, it is necessary to design the sensors so that their resonant frequencies exceed the frequencies of the seismic oscillations studied. Nevertheless, in order to improve the measurement accuracy, it is appropriate to adjust the results obtained by taking into account the sensors frequency response. This can be done by using the method described in the paper to calculate the values of $\gamma_{i}$ and $S\left(f_{i}\right)$ for the respective frequency range.

\section{ACKNOWLEDGEMENT}

This study was performed under contract No KP - 06 - RUSSIA/23 and 19-5718004 dated September 28, 2019 between IMSETHC-BAS and R-Sensors for financing a research project for the implementation of the project "Study of the amplitude frequency characteristics of seismic signals generated by surface sources," funded by designated based financing conducted by Fund "Science" "Competition for projects under programs of bilateral cooperation in 2018 - Bulgaria - Russia 2018-2019"

\section{REFERENCES}

[1] D. Dimitrov, V. Panevski, G. Nikolov (2016) Example of security management system of the organization - components and concepts. International Scientific Journal “Science. Business. Society” 1(4) 37-40.

[2] S. Petkov, V. Nikolova, P. Petkov, S. Yaneva (2018) Main directions for the Development of Protection Equipment of Dynamic Type Using Electrical Energy. European Journal of Engineering Research and Science 3(5) 55-59, DOI: http:/dx.doi.org/10.24018/ejers.2018.3.5.730.

[3] P. Vasileva (2019) Terrorist Chemical, Biological, Radiological and Nuclear Threat to Europe. International Scientific Journal "Security \& Future" 3 93-104.

[4] V. PANeVski (2018) Integrated critical infrastructure security management. International Scientific Journal "Security \& Future" 2(1) 3-6.

[5] V. PANeVsKi (2019) Competence centres and intelligent security systems in Bulgaria. International Scientific Journal "Security \& Future" 3 (1) 14-16.

[6] V. PANeVski (2019) Systemic approach to the development of security systems for critical infrastructure protection as a research methodology applied at the center of competence Quasar. International Scientific Journal "Security \& Future" 3(4) 144-147.

[7] B. Genov, R. Ganev, V. Grozev, Pl. Tchernokojev (2008) Acoustic emission monitoring of penetration and perforation occurring under impact loading caused by bullets with segmented and monolithic cores. 7th International Armament Conference, Pultusk 8-10.10.2008, Poland, In Scientific Aspects of Armament and Safety Technology I Chapter 4 471-475. 
[8] B. Genov, D. Nedelchev, M. Mihovski, Y. Mirchev (2019) Comprehensive approach for service life assessment of solid-propellant rocket motors. NDT Days II(4) 467-475.

[9] B. Genov, N. Vulkov, G. Genov, Pl. Chernokojev (2004) Research of the influence of the type jacket of the bullet of the test ammunitions at the testing of the aramid materials. ISSN: 1312-2916 (print) 269-276.

[10] B. Genov, D. Kirkov, M. Mihovski, M. Mirchev (2018) Ageing of solid rocket propellants investigated by ultrasound technique. NDT Days I(5) 577-582.

[11] G. Asch ET AL. (1991) "Les capteurs en instrumentation industrielle". Dunod edition, Paris.

[12] D. Zaitsev, V. Agafonov, I. Evseev (2018) Study of Systems Error Compensation Methods Based on Molecular-Electronic Transducers of Motion Parameters. Journal of Sensors 2018, Article ID 6261384, 9 pages.

[13] A. Bugaev, V. Agafonov, A. Antonov, K. Belotelov, P. Dudin, E. Egorov, I. Egorov, T. Krishtop, A. Neeshrapa, V. Popov, A. Shabalina, S. Vergeles, V. Uskov, D. Zaytsev, D. Zhevnenko, S. Zhabin, V. Krishtop (2018) Molecular electronic transducers for measuring instruments. Journal of Communications Technology and Electronics 63(12) 1339-1351.

[14] I. Evseev, D. Zaitsev, V. Agafonov (2019) Study of Transfer Characteristics of a Molecular Electronic Sensor for Borehole Surveys at High Temperatures and Pressures. Sensors 192545.

[15] D. Zaitsev, E. Egorov, M. Ryzhkov, G. Velichko, V. Gulenko (2019) Lowfrequency, low-noise molecular-electronic hydrophone for offshore and tranzit zone seismic exploration. Conference proceedings of $19^{\text {th }}$ Int. Multidisciplinary Scientific Geoconference \& Expo SGEM 2019, June 28 - July 7, Albena, Bulgaria 19 961-968. 